\title{
Establishment of a rhabdomyosarcoma xenograft model in human-adapted mice
}

\author{
GUIDO SEITZ $^{1 *}$, MATTHIAS PFEIFFER ${ }^{2 *}$, JÖRG FUCHS $^{1}$, STEVEN W. WARMANN $^{1}$, IVO LEUSCHNER $^{3}$, \\ CHRISTIAN VOKUHL ${ }^{3}$, PETER LANG ${ }^{2}$, RUPERT HANDGRETINGER ${ }^{2}$ and SORIN ARMEANU-EBINGER ${ }^{1}$ \\ Departments of ${ }^{1}$ Pediatric Surgery and ${ }^{2}$ Pediatric Oncology/Hematology, University Children's \\ Hospital, Tübingen; ${ }^{3}$ Institute of Pathology, University Hospital Schleswig-Holstein, Kiel, Germany
}

Received April 21, 2010; Accepted June 16, 2010

DOI: $10.3892 /$ or_00000956

\begin{abstract}
The outcome of patients with advanced stage rhabdomyosarcoma (RMS) is still sobering. This outcome has not improved through conservative treatments. Therefore, novel treatment approaches such as immunotherapy need to be evaluated in human-adapted animal models. The aim of this study was to develop a humanized mouse model of childhood RMS as a basis for the study of immunotherapeutic approaches. Therefore, NOD/LtSz-scid IL2 $r \gamma^{\text {null }}$ mice were used for all the experiments $(n=19)$. The animals underwent sublethal irradiation on days 1 and 2 ( 1 x 300 cGy). After irradiation, the transplantation of human CD34+-cells $(1,000,000$ cells per animal i.v.) was carried out. Five animals served as the control and did not undergo stem cell transplantation. The engraftment of human cells was assessed in peripheral blood on days 21 and 55 by FACS analysis. Eight weeks after transplantation, the subcutaneous xenotransplantation of human alveolar and embryonal RMS cell lines was carried out. Tumor growth was monitored and tumors were resected 93 days after $\mathrm{CD} 34^{+}$-transplantation. The tumor specimens were evaluated histologically. The successful engraftment of human cells with the establishment of a human immune system was observed in 12 out of 14 animals. $\mathrm{B}$ and $\mathrm{T}$ cells were mostly detected in the peripheral blood. There were only a few monocytes and almost no natural killer cells. The xenotransplantation of alveolar RMS resulting in subcutaneous tumor growth was feasible in 7 animals. The xenotransplantation of embryonal RMS was performed in 5 animals and led to tumor growth in 1 animal. A histological work up showed either alveolar or embryonal RMS cells with
\end{abstract}

Correspondence to: Dr Guido Seitz, Department of Pediatric Surgery, University Children's Hospital, Hoppe-Seyler-Strasse 3, 72076 Tübingen, Germany

E-mail: guido.seitz@med.uni-tuebingen.de

*Contributed equally

Key words: rhabdomyosarcoma, xenograft, human-adapted mice central necrosis. This is the first time a xenotransplantation model of human RMS has been developed in a humanized mouse model. The establishment of subcutaneous tumor xenografts was more effective in the alveolar subtype. This model offers a basic tool for further analyzing novel immunotherapeutic approaches in RMS, and could possibly be used in other solid pediatric tumors.

\section{Introduction}

Rhabdomyosarcoma (RMS) is the most common pediatric soft tissue sarcoma and represents $7-8 \%$ of all the solid malignant tumors in childhood (1). Patient's prognosis depends on the primary tumor localization, histological subtype, stage of disease, and the age at diagnosis (2-4). Despite treatment efforts in localized disease, in which survival rates have improved from 25 to $70 \%$ in the last decades (5), patients with metastatic disease still have a poor outcome (6). Conventional treatment approaches including different chemotherapy regimens have not led to the designated treatment success, as the 5-year OS of patients with metastasized RMS was only $27 \%$ in large clinical trials (7).

Therefore, other treatment modalities are required for children with advanced stage RMS. Immunotherapy seems to be a promising novel treatment approach (8). In RMS, a number of immunotherapeutic treatment approaches have been described, such as autologous T cells, influenza vaccinations, and dendritic cells (8). Additionally, haploidentical stem cell transplantation seems to be a promising approach (9). Besides the in vitro evaluation of immunotherapeutic approaches, there is a lack of sufficient animal models of human RMS, especially in animals with a human-adapted immunosystem. Therefore, the aim of this study was to establish a reproducible animal model of RMS xenografts in a mouse model with a human-adapted immunosystem.

\section{Materials and methods}

Animals. Eight-week-old NOD/LtSz-scid IL2r $\gamma^{\text {null }}$-mice, weighing 25 to $30 \mathrm{~g}$, were used for all the experiments. This mouse strain was initially described and established by Shultz et al (10). These animals were chosen as they have severe defects of innate immunity such as the lack of mature $\mathrm{T}$ and $\mathrm{B}$ cells, and natural killer (NK) cells. Additionally, 
these animals do not develop thymic lymphomas (10). The animals were obtained from Jackson Laboratories (Bar Harbor, ME, USA) as well as from our own laboratory. A total number of 19 animals were used for all the experiments. The animals were kept under pathogen-free conditions, fed an autoclaved standard diet and given free access to sterilized water. All animal experiments were approved by the local government ethics committee for animal studies (Regierungspräsidium Tübingen, CK 01/06).

Cell lines and culture conditions. The embryonal RMS (RD) cell line (ATCC, USA) and the alveolar RMS (Rh30) cell line (DSMZ, Braunschweig, Germany) were cultured in DMEM medium supplemented with $10 \%$ fetal calf serum, $4.5 \% \mathrm{~L}$ Glu and $2.5 \%$ HEPES in a humified atmosphere containing $5 \% \mathrm{CO}_{2}$ at $37^{\circ} \mathrm{C}$. All cells were mycoplasma-negative.

Transplantation of mobilized human hematopoietic stem cells. The transplantation of human hematopoietic stem cells (HSC) was performed as described previously (10). The objective of HSC transplantation in these animals is the development of human $\mathrm{CD}^{+}, \mathrm{CD}^{+}$and $\mathrm{CD}^{+}{ }^{+} \mathrm{CD} 8^{+} \mathrm{T}$ cells, as well as $\mathrm{Ig}^{+} \mathrm{B}$, myeloid and $\mathrm{NK}$ cells (10). In brief, the engraftment was conducted with HSC from individual donors. Therefore, peripheral blood cells from healthy donors were obtained after G-CSF administration [10 g/kg/day s.c. injection (Neupogen; Amgen, Thousand Oaks, CA, USA) for 4 days]. A single leukapheresis was performed on day 5. Written consent was obtained from all the donors and the study was approved by the local ethics committee (89/2007V). $\mathrm{CD} 34^{+}$cells were selected using the Miltenyi CliniMACS system (11). The purity of the CD34+ cells was $>90 \%$. Cells $\left(10^{7}\right)$ were prepared for stem cell transplantation. A total number of 14 animals underwent sublethal irradiation with $2 \times 150 \mathrm{cGy}$, cesium 137 on days 1 and 2. Five animals served as the controls and received no transplantation or irradiation. Subsequently, CD $34^{+}$-cells $\left(10^{6}\right.$ cells $)$ were injected i.v. into the tail vein of the animals. Mice received Fc-IL7 fusion protein (EMD Lexigen Research Center, Billerica, MA, USA) injected into the tail vein at a dose of $20 \mathrm{~g}$ once a week starting 1 week after the CD34+-transplantation. Treatment with Fc-IL7 was continued until the animals were sacrificed. The engraftment of the human cells was assessed on days 21 and 55 after the CD34+-injection using FACS analysis of the peripheral blood, drawn from the retrobulbar venous plexus of the animals.

Xenotransplantation of human RMS. In order to have comparable tumor cells, only passages 2-4 were used for the xenotransplantation. Tumor cells were grown as described above. The cells were trypsinized and resuspended in RPMI1640 medium (Gibco, Berlin, Germany). Approximately $10^{6}$ tumor cells were injected into the right flank of the animals 50 days after stem cell transplantation. Cells from the Rh30 cell line were transplanted into 7 mice and those from the RD cell line were transplanted into 5 animals. Five animals without stem cell transplantation underwent xenotransplantation with Rh30 cells and served as the controls. The transplanted animals were continuously observed and clinically examined. Tumor volumes were measured every 5 days with a caliper rule (a, length; $b$, width; $c$, height). Tumor volumes were calculated using the formula $\mathrm{a} / 2 \mathrm{x} \mathrm{b} / 2 \mathrm{x} \mathrm{c} / 2 \mathrm{x}$ $4 / 3 \pi$. The animals were sacrificed 93 days after the CD $34^{+}-$ transplantation and the tumors were resected for further histological analysis. For comparison with our previously described xenograft model with NMRI nu/nu mice, we used tissue samples from subcutaneous growing Rh30 xenografts $(\mathrm{n}=6)(12)$.

Histological analysis. The tissue was fixed in $37 \%$ formalin and processed for further histological analysis. Therefore, tissue processing was continued in a vacuum tissue processor (Leica TP 1050, Leica Wiesloch, Germany) and the tissue was paraffin embedded after tissue processing. Sections of $3 \mu \mathrm{m}$ were cut and the tissue was deparaffinized with xylol and ethanol (Roth, Karsruhe, Germany). A standard hematoxylin and eosin staining was carried out for the evaluation of necrosis and histological changes. For immunohistochemistry, antigen retrieval was performed by heat application in citrate buffer as recommended for the antibodies to Desmin (D33; Dako, Glostrup, Denmark). Slices were blocked with Tris-buffered saline with $0.2 \%$ Tween-20 and $1 \%$ FCS and incubated overnight at $4{ }^{\circ} \mathrm{C}$ with Mouse anti-Desmin (1:250). The following day, antibody binding was revealed with Vectastain Universal Elite ABC Kit (Linaris GmbH Wertheim-Bettingen, Germany) and DAB solution (Dako GmbH, Hamburg, Germany) was used as a substrate. Nuclear and cytoplasmic structures were stained with hematoxylin and eosin. The slides were then analyzed using 10 high power fields (400x).

Cell proliferation. In order to evaluate cell proliferation, a Ki-67 immunohistochemistry assay was carried out. Therefore, the slides were rinsed in PBS for 5 min. Blocking was performed using 1.5\% normal goat serum (\#X0907, Dako, Glostrup, Denmark) for $30 \mathrm{~min}$ followed by the monoclonal mouse anti-human Ki-67-primary antibody-clone MIB-1 (\#M7240, Dako Cytoformation, Glostrup, Denmark; dilution 1:100) for $60 \mathrm{~min}$ at room temperature. The slides were rinsed for 3 x 5 min in PBS. A FITC-conjugated secondary goat anti-mouse antibody (\#115-095-062, Dianova, Hamburg, Germany; dilution 1:100) was added for $30 \mathrm{~min}$ at room temperature, followed by a PBS washing step ( 3 x 5 min). For counterstaining of the nucleus, DAPI was used for $1 \mathrm{~min}$ (\#D-9452, Sigma-Aldrich; dilution 1:10000) followed by a PBS washing step. The slides were mounted and analyzed by fluorescence microscopy.

Flow cytometry. Blood cells were incubated with erythrocyte lysis buffer (Becton-Dickinson, Heidelberg, Germany) and with prestained antibodies to CD3-PerCP, CD14-FITC, CD19-APC, CD56-PE and CD45-PE-Cy7 (all from BectonDickinson), or with the respective isotype controls. The cells were analyzed on an LSR II cytometer with FACSDiva software (Becton-Dickinson).

Statistical analysis. Statistical analysis was performed by one-way ANOVA and ranks and Student's t-test. All numerical data are expressed as mean \pm SEM. Significance was assumed for all $\mathrm{p}<0.05$. 
A

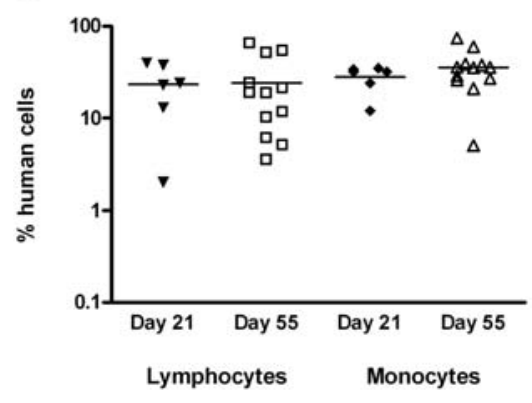

B

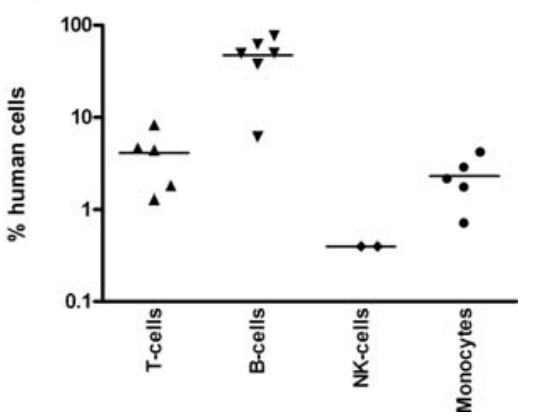

Figure 1. (A) Engraftment of human cells in animals undergoing CD34+-transplantation. There was no significant difference between days 21 and 55 after transplantation. (B) Distribution of human leukocytes and lymphocytes on day 55 after transplantation.

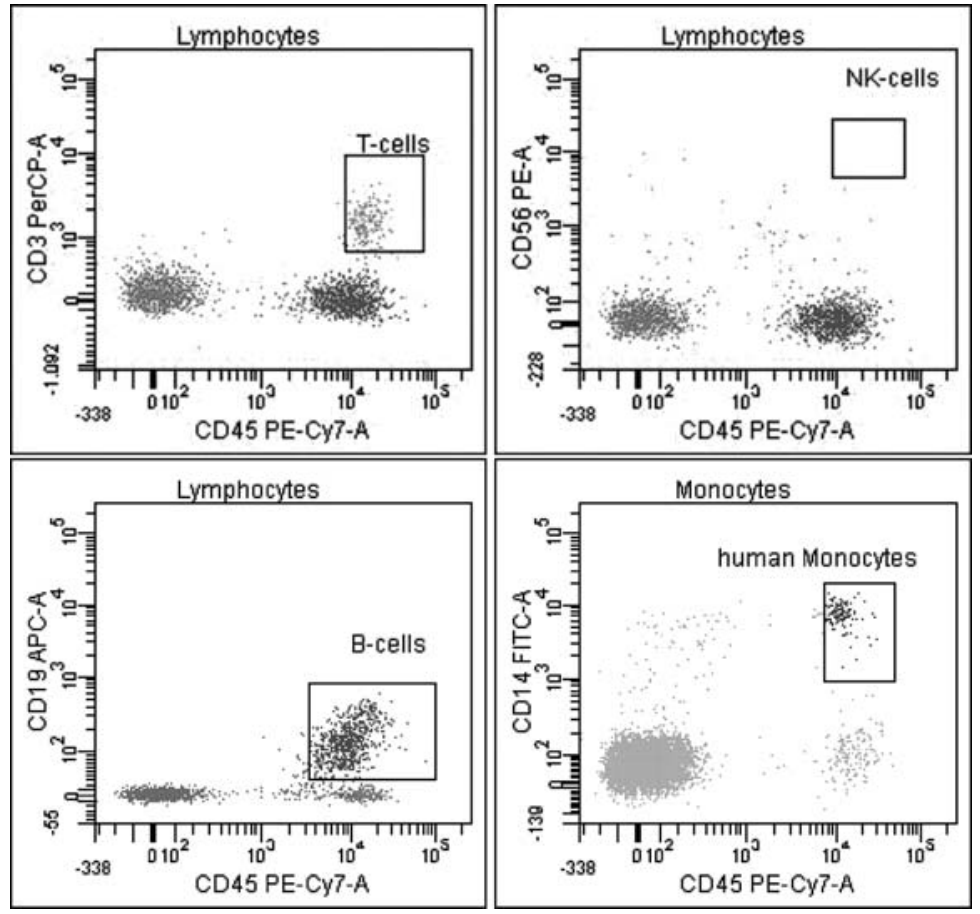

Figure 2. FACS analysis showing the successful engraftment of human HSC in mice on day 55.

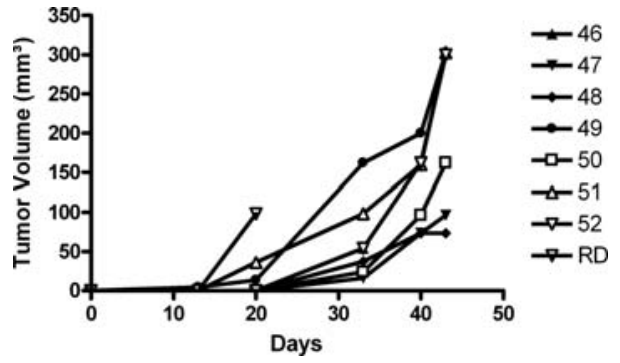

Figure 3. Growth curves of human Rh30 xenografts (the numbers represent the different animals).

\section{Results}

Engraftment of human HSC. Twenty-one days after the transplantation with $\mathrm{CD}_{3} 4^{+}$-cells, we found human $\mathrm{B}$ and $\mathrm{T}$ cells as well as human monocytes in the peripheral blood of the animals, thus proving the successful engraftment of human HSC in 12/14 animals (Fig. 1). On day 55, the number of CD45+-cells, used as a marker for all human leukocytes, did not change significantly (Fig. 1). In 2 animals, there was no evidence of human HSC and therefore no adequate engraftment of HSC was found. These animals were excluded from further analyses. In the animals with a successful engraftment, we mostly found $\mathrm{B}$ and $\mathrm{T}$ cells. There was a low amount of monocytes and $<0.4 \%$ NK cells in the peripheral blood. FACS analysis revealed the successful engraftment of human HSC in mice (Fig. 2). Analysing the leucocytes, we found 55 days post-transplantation a mean amount of $24 \pm 6 \%$ of human lymphocytes and $35 \pm 5 \%$ of human monocytes (Fig. 1). The mean amount of human $\mathrm{T}$ cells was $4 \pm 1 \%$, human B cells $47 \pm 10 \%$ and nearly no NK cells were detectable.

Establishment of human RMS xenografts. Subcutaneous xenotransplantation with Rh30 cells led to the development of subcutaneous xenografts in $7 / 7$ animals. Tumors were 


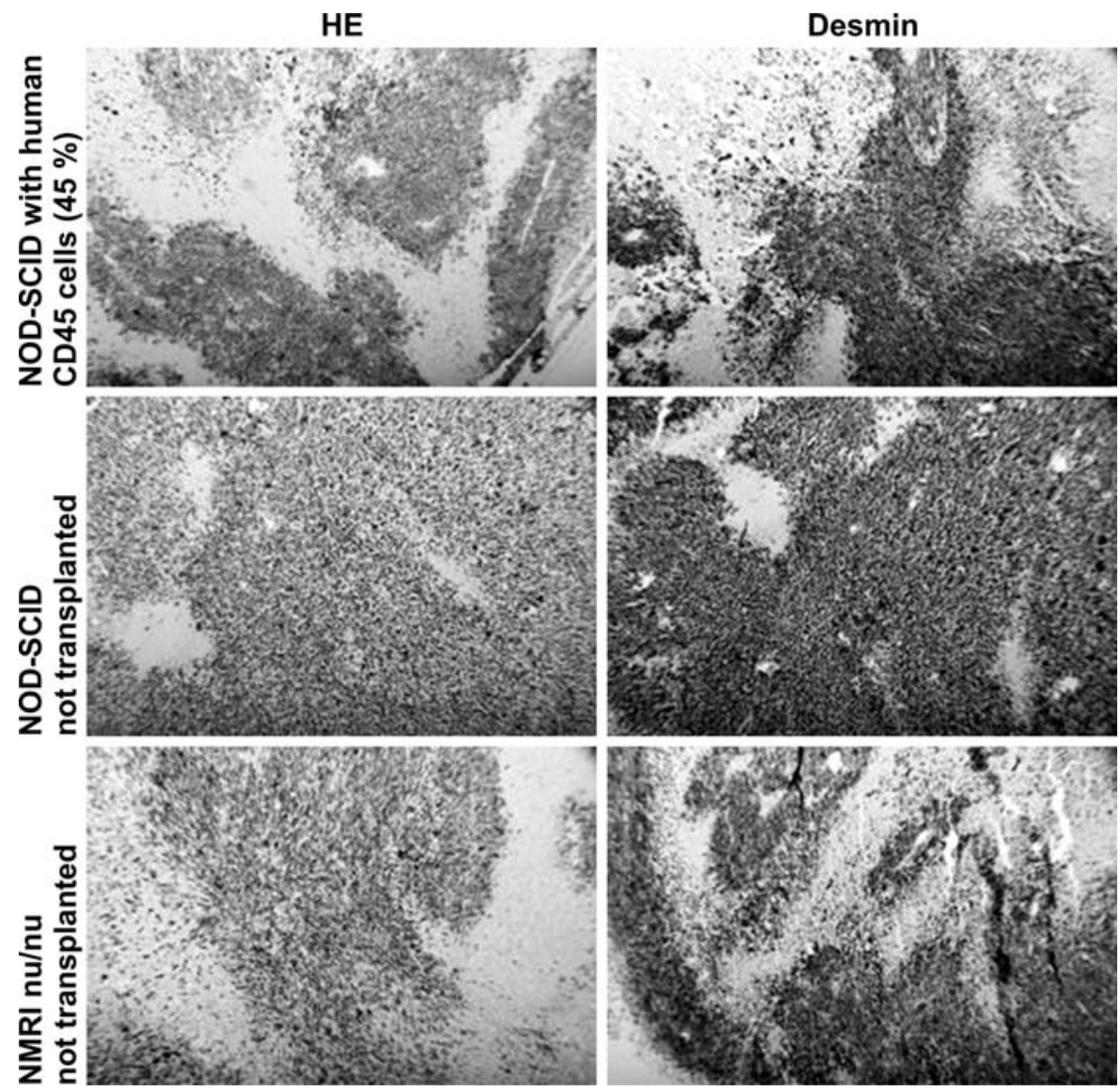

Figure 4. Cell morphology (hematoxylin and eosin staining) and Desmin immunohistochemistry in Rh30 tumors (400x). The extent of necrosis did not differ significantly between the animals undergoing CD34+-transplantation, those without CD34+-transplantation and the NMRI nu/nu mice.

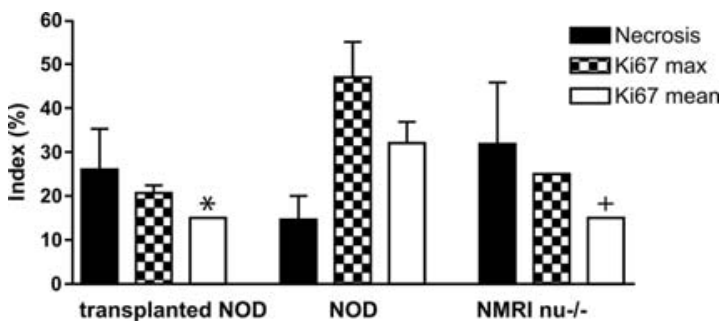

Figure 5. Cell proliferation of Rh30 tumors in animals undergoing CD34+transplantation (*vs. animals without CD34+-transplantation), those without CD34+transplantation (+, p<0.05 vs. NMRI nu/nu) and the NMRI nu/nu mice.

macroscopically detectable 10 to 20 days after xenotransplantation and reached a mean tumor volume of $220 \pm 40 \mathrm{~mm}^{3}$ within 3 weeks of the initial appearance (Fig. 3). After xenotransplantation with the RD cells, 3 animals died 1 week after xenotransplantation for other reasons. In the 2 surviving animals, a xenograft could only be established in 1 animal.

Histological workup. A standard histology of the xenotransplants revealed highly malignant RMS of the embryonal or alveolar subtype according to the initially transplanted tumor cells. All the tumor xenografts were Desmin-positive. In the Rh30 xenografts of the NOD mice undergoing stem cell transplantation, the extent of necrosis ranged from $2-70 \%$ (mean, $23 \pm 9 \%$ ). In the xenografts of the NOD mice without stem cell transplantation, the extent of necrosis ranged from $3-30 \%$ (mean, $15 \pm 5 \%$ ) and in the NMRI nu/nu mice from $3-80 \%$ (mean, $31 \pm 14 \%, \mathrm{p}=0.56$ vs. other groups). The histological findings are shown in Fig. 4.

Cell proliferation. The mean cell viability in the xenografts of animals with Rh30 undergoing stem cell transplantation was $16 \pm 1 \%$ ( $p<0.05$ vs. NOD mice without stem cell transplantation). In the tumors from the animals without stem cell transplantation it was $32 \pm 5 \%$ ( $p<0.05$ vs. NMRI nu/nu mice). In the xenografts of the NMRI nu/nu mice the mean cell viability was $15 \pm 0 \%$ (Fig. 5). There was no significant difference between the NOD mice after stem cell transplantation and the NMRI nu/nu mice.

\section{Discussion}

Novel experimental immunotherapeutic approaches for advanced stage RMS have been identified as a possible way to improve the outcome of affected patients. Investigations in humans are limited by technical and ethical considerations. Therefore, alternative disposable and reproducible animal models are desirable. Mouse models are regarded as sufficient tools for immunological studies. However, they cannot accurately predict the outcome in humans (13). Consequently, mouse models with the establishment of a human-adapted immune system have been introduced. So far, no such model exists for the study of immunotherapy against RMS. 
In the past 20 years, many humanized animal models have been described (14). Today, the two most commonly used models are the BALB/c-Rag2 $2^{\text {null }}$ IL2r $\gamma^{\text {null }}$-mouse (15) and the NOD/LtSz-scid IL2r $\gamma^{\text {null }}$-mouse model (10). After the xenotransplantation of human HSC, there is an engraftment of the transplanted cells with the consecutive production of all human myeloid and lymphoid cell lineages. There seems to be an advantage in the use of newborn animals compared to adult animals, as newborns do not require the exogenous supply of human IL-7 for sustained thymopoiesis and have a better bone marrow chimerism (13). Both animal models could be used for hematopoietic reconstitution, microbial infections as well as vaccine development (13).

We initially used adult HSCs and BALB/c-Rag2 $2^{\text {null }}$ IL $2 r \gamma^{\text {null }}$-mice for our experiments. However, the engraftment rates did not exceed $10 \%$. Additionally, we found no specific $\mathrm{T}, \mathrm{B}$, or NK cell markers (unpublished data). These observations could be due to the fact that we had to use adult HSCs instead of cord blood HSCs due to ethical limitations. Therefore, we decided to switch to the adult NOD/LtSz-scid IL2 $r \gamma^{\text {null }}-$ mouse model described by Shultz et al (10).

We found a successful engraftment in $85 \%$ of the animals with human myeloid and lymphoid lineages. The amount of human cells was between $4 \%$ in the T cells and $47 \%$ in the B cells. Of all the leukocytes, $24 \%$ were human lymphocytes and $35 \%$ were human monocytes. We found only very few NK cells. Similar to the initial description of the model by Shultz et al (10), only a few NK cells were detected in the peripheral blood. Most of the NK cells were found in the bone marrow. Recently, Huntington et al showed that exogenous human IL-15 or IL-15-IL-15-R $\alpha$ complexes, are required for further NK development in $\mathrm{Rag} 2^{-/ \gamma} \mathrm{\gamma}^{-/-}$mice transplanted with human HSC (16). The lack of sufficient amounts of human IL-15 could be the reason for the low NK cell numbers in the NOD/LtSz-scid IL2 $2 \gamma^{\text {null }}-$ mice. The engrafted mice generate antibodies following the immunization with $\mathrm{T}$ cell-dependent antigens. The ability of these HSC humanized immunodeficient NOD/LtSz-scid IL2r $\gamma^{\text {null }}$ mice to reject human allografts has not yet been investigated (10).

Several different tumors were engrafted quite efficiently in the NOD/LtSz-scid IL2r $\gamma^{\text {null }}$-mice (http://jaxmice.jax.org/ strain/005557.html), given that the development of the murine immune system is impaired as in the CB17-scid mice. These animals did not undergo a CD34+-transplantation for the establishment of a human-adapted immune system. This mouse strain is deficient in mature lymphocytes, serum Ig's are not detectable and NK cell cytotoxic activity is extremely low. In our study, the establishment of human RMS xenografts was feasible in all animals receiving $\mathrm{Rh} 30$. In RD, only 1 xenograft could be established. A possible explanation is that Rh30s are biologically more aggressive, show better growth in vitro, and are generally more potent in generating xenografts compared to the RDs (12). It should be noted that, there was no general rejection of the xenografts by the human immune system, although circulating human $\mathrm{T}$ and $\mathrm{B}$ cells were present before challenging the animals with tumor cells. This may be a result of the lack of innate immunity like NK cells, as first reaction against the tumor. These findings are comparable to a study by Simpson-Abelson et al on NOD/
LtSz-scid IL2r $\gamma^{\text {null }}$-mice with the successful xenotransplantation of human lung tumor tissue concomitant with the expansion of tumor resident human $\mathrm{T}$ cells. However, in contrast to our study, there was no establishment of a human adapted immune system in these animals (17).

The extent of necrosis was not significantly different in the animals undergoing the $\mathrm{CD} 34^{+}$-transplantation compared to the controls without the $\mathrm{CD} 34^{+}$-transplantation and compared to the NMRI nu/nu mice. However, there was a trend towards higher necrosis rates in the $\mathrm{CD} 34^{+}$-transplanted NOD/LtSz-scid IL2r $\gamma^{\text {null }}$-mice compared to the untransplantated animals. This could be caused by the interaction with the human-adapted immune system. In the NMRI nu/nu mice, comparable necrosis rates were found as in the $\mathrm{CD} 34^{+}-$ transplanted NOD/LtSz-scid IL2 $\gamma^{\text {null }}$-mice. The immune system of the NMRI nu/nu mouse strain is characterized by a small population of $\mathrm{T}$ cells. A compensatory increase in the NK cell level was observed in comparison to the normal $\mathrm{BALB} / \mathrm{cA}$ mice and this could be responsible for the extent of tumor necrosis (18). Another aspect is that the extent of necrosis could depend on the tumor volume and is not influenced by the human immune system.

The mean cell viability of the RMS xenografts was significantly lower in the animals undergoing CD34+-transplantation compared to the animals without $\mathrm{CD} 34^{+}$-transplantation. The human immune cells could have an inhibitory influence on the cell viability in these animals. Additionally, the cell viabilty was similar to our observation in the NMRI nu/nu mice. This could be caused by the fact that these animals have a partial immunity through the NK cells possibly influencing cell viability. In the NOD/LtSz-scid IL2r $\gamma^{\text {null }}$ mice without $\mathrm{CD} 34^{+}$-transplantation, there was no sufficient immune response and therefore, cell viability could be higher than in the other groups.

In conclusion, this is the first time an animal model of RMS xenografts has been generaed using NOD/LtSz-scid IL2 $r \gamma^{\text {null }}$-mice with a human-adapted immune system. This model was more successful in alveolar RMS than in embryonal RMS. This model will help to evaluate immunotherapeutic approaches in vivo in this tumor entity.

\section{References}

1. Crist WM, Anderson JR, Meza JL, et al: Intergroup rhabdomyosarcoma study IV: results of patients with non-metastatic disease. J Clin Oncol 19: 3091-3102, 2001.

2. Cocker HA, Pinkterton CR and Kelland LR: Characterization and modulation of drug resistance of human rhabdomyosarcoma cell lines. Br J Cancer 83: 338-345, 2000.

3. Newton WA Jr, Gehan EA, Webber BL, et al: Classification of rhabdomyosarcomas and related sarcomas. Cancer 76: 1073-1085, 1995.

4. Lawrence W Jr, Anderson JR, Gehan EA and Maurer H: Pretreatment TNM staging of childhood rhabdomyosarcoma: a report of the Intergroup study group. Children's cancer study group. Pediatric Oncology Group. Cancer 80: 1165-1170, 1997.

5. Klingebiel T, Boos J, Beske F, et al: Treatment of children with metastatic soft tissue sarcoma with oral maintenance compared to high dose chemotherapy: report of the HD CWS-96 trial. Pediatr Blood Cancer 50: 739-745, 2008.

6. Koscielniak E, Harms D, Henze G, et al: Results of treatment for soft tissue sarcoma in childhood and adolescence: A final report of the German Cooperative Soft Tissue Sarcoma Study CWS-86. J Clin Oncol 17: 3706-3719, 1999. 
7. Weber G, Karbach J, Kuci S, et al: WT1 peptide-specific T cells generated from peripheral blood of healthy donors: possible implications for adoptive immunotherapy after allogeneic stem cell transplantation. Leukemia 23: 1634-1642, 2009.

8. Mackall CL, Rhee EH, Read EJ, et al: A pilot study of consolidative immunotherapy in patients with high-risk pediatric sarcomas. Clin Cancer Res 14: 4850-4858, 2008.

9. Lang P, Pfeiffer M, Müller I, et al: Haploidentical stem cell transplantation in patients with pediatric solid tumors: preliminary results of a pilot study and analysis of graft versus tumor effects. Klin Padiatr 218: 321-326, 2006.

10. Shultz LD, Lyons BL, Burzenski LM, et al: Human lymphoid and myeloid cell development in NOD/LtSz-scid IL2R gamma null mice engrafted with mobilized human hemopoietic stem cells. J Immunol 174: 6477-6489, 2005.

11. Schumm M, Lang P, Taylor S, et al: Isolation of highly purified autologous and allogeneic peripheral CD34+ cells using CliniMACS device. J Hematother 8: 209-218, 1999.

12. Seitz G, Warmann SW, Vokuhl C, Heitmann H, Treuner C, Leuschner I and Fuchs J: Effects of standard chemotherapy on tumor growth and regulation of multidrug resistance genes and proteins in childhood rhabdomyosarcoma. Pediatr Surg Int 23 431-439, 2007.
13. Macchiarini F, Manz MG, Palucka AK and Shultz LD: Humanized mice: are we there yet? J Exp Med 202: 1307-1311, 2005.

14. Manz MG and Di Santo JP: Renaissance for mouse models of human hematopoiesis and immunobiology. Nat Immunol 10: 1039-1042, 2009

15. Traggiai E, Chicha L, Mazzucchelli L, Bronz L, Piffaretti JC, Lanzavecchia A and Manz MG: Development of a human adaptive immune system in cord blood cell-transplantated mice. Science 304: 104-107, 2004

16. Huntington ND, Legrand N, Alves NL, et al: IL-15 promotes trans-presentation promotes human NK cell development and differentiation in vivo. J Exp Med 206: 25-34, 2009.

17. Simpson-Abelson MR, Sonnenberg GF, Takita H, et al: Longterm engraftment and expansion of tumor-derived memory $\mathrm{T}$ cells following the implantation of non-disrupted pieces of human lung tumor into NOD/LtSz-scid IL $2 \mathrm{r} \gamma^{\text {nulf }}$ mice. J Immunol 180: 7009-7018, 2008.

18. Hasui M, Saikawa Y, Miura M, et al: Effector and precursor phenotypes of lymphokine-activated killer cells in mice with severe combined immunodeficiency (scid) and athymic (nude) mice. Cell Immunol 120: 230-239, 1989. 